\title{
Reconstruct the Mesoscale Information of Typhoon with BDA Method Combined with AMSU-A Data Assimilation Method
}

\author{
Yunfeng Wang, ${ }^{1,2}$ Haiyang Zhang, ${ }^{1}$ Bin Wang, ${ }^{2}$ Yueqi Han, ${ }^{1}$ and Xiaoping Cheng ${ }^{1}$ \\ ${ }^{1}$ Laboratory of Military Meteorology, Institute of Meteorology, PLA University of Science and Technology, Nanjing, 211101, China \\ ${ }^{2}$ LASG, Institute of Atmospheric Physics, Chinese Academy of Sciences, Beijing 100029, China
}

Correspondence should be addressed to Yunfeng Wang, wangyf@mail.iap.ac.cn

Received 28 December 2009; Revised 24 March 2010; Accepted 20 April 2010

Academic Editor: Zhaoxia Pu

Copyright () 2010 Yunfeng Wang et al. This is an open access article distributed under the Creative Commons Attribution License, which permits unrestricted use, distribution, and reproduction in any medium, provided the original work is properly cited.

\begin{abstract}
This paper conducts the assimilating experiments and simulating experiments on typhoon "Aere" (No. 0418), by use of bogus data assimilation (BDA) method combined with advanced microwave sounding unit-A (AMSU-A) data assimilation method in the fifth-generation National Center for Atmospheric Research (NCAR)/Penn State Mesoscale Model Version-3 (MM5V3), the Radiative Transfer for TIROS-N Operational Vertical Sounder Version-7 (RTTOV) model, and their adjoint models. The Bogus data constructed with BDA technique are mainly located at sea level, while the peak energy contribution levels of the sounder channels selected in AMSU-A data assimilation technique are mainly located at upper troposphere. The two types of data can reconstruct the meso-scale information and improve the typhoon initial fields under the model dynamic forcing effect, respectively from the low level and the upper level of atmosphere during the assimilating process. Numerical results show that with fourdimensional variational data assimilation (4DVAR) technique the circulation of initial fields is improved, the "warm core" of typhoon is enhanced, the "cloud water" phenomenon that occurs in the optimal initial fields and the numerical model is changed into "warm start" from "cold start".
\end{abstract}

\section{Introduction}

Typhoon is one of the most frequent disasters affecting human beings. With the development of numerical forecasting techniques, numerical forecasting of typhoon has entered an operational stage. However, the prediction accuracy is far from meeting the requirements of disaster prevention and reduction. If the track and intensity of typhoon can be accurately forecasted, necessary preparedness can be performed beforehand and serious economic loss can be much reduced. Due to severe deficiency of conventional observation data over sea, the results of objective analysis can not precisely describe the thermal structure and circulation characteristics of initial typhoon, especially its mesoscale structure, which is one of the main reasons for serious errors in typhoon numerical forecasting. Therefore, how to provide more rational initial values is an urgent task.

Since 1990s, an initializing method of artificial typhoon mode is introduced into typhoon forecasting research [1$3]$. With this method, an ideal bogus typhoon mode with 3-dimensional circulation structure and thermal structure is constructed according to the observation data, and then it is implanted into the initial typhoon analysis fields to construct new initial typhoon optimal fields. This method is now widely applied in Typhoon Numerical Prediction Operational System in many countries, which improves the typhoon numerical forecast skill greatly. However, there are still a lot of shortcomings in this method.

The concept of 4DVAR was initially put forward and then developed by Lewis and Derber [4], Le Dimet and Talagrand [5], and Courtier and Talagrand [6]. Later, with 4DVAR technique, a new method to optimize the rough initial fields of tropical cyclone was proposed $[7,8]$, which is named bogus data assimilation (BDA) method, that is, with 4DVAR technique, through assimilating the sea level pressure of ideal vortex and gradually adjusting the model background field, more actual vortex with dynamical and physical balance is obtained. Numerical experiments on hurricane "Felix" show that this method effectively improves the characteristics of hurricane eye and forecasting of hurricane intensity. 
Through numerical simulation of hurricane Georges and Bonnie, $\mathrm{Pu}$ and Braun [9] thought that assimilating the wind data of bogus vortex was also important to the adjustment of initial field. A hybrid variational scheme (HVAR) is developed by Weng et al. [10] and it was applied in two hurricane cases, resulting in improved analyses of three-dimensional structures of temperature and wind fields as compared to operational model analysis fields. It is found that HVAR reproduces detailed structures for the hurricane warm core at the upper troposphere. Both lowerlevel wind speed and upper-level divergence are enhanced, with reasonable asymmetric structure. With the weather research and forecasting three-dimensional variational data assimilation system (WRF 3D-VAR), it is indicated that hurricane initialization with the BDA technique can improve the forecast skills of track and intensity in the Advanced Research WRF (ARW) [11]. Besides, many other researchers also have done much work on studying the BDA method $[12,13]$.

With the rapid development of satellite observing system, the satellite remote sensing data gradually becomes one of the major observation data sources due to its wide coverage and high-spatial and -temporal resolution; the assimilation of satellite data also turns to be an important instrument to reconstruct the mesoscale structure of typhoon. The fast radiative transfer modeling and its components, critical for satellite data assimilation, are summarized and discussed for their potential applications in operational global data assimilation systems [14]. Since the microwave radiation can pass through deep cloud layer and its brightness temperature data can well indicate the vertical structure of atmosphere under cloud, it is very useful to improve typhoon circulation structure and determinate typhoon initial location.

Zou et al. [15] successfully assimilated Geostationary Operational Environmental Satellite (GOES) brightness temperature data into the initial field of typhoon by use of the 4DVAR technique in their study of the hurricane on the east coast of America. The results indicated that the forecasting of typhoon intensity, track and precipitation could be greatly improved. Le Marshall et al. [16] assimilated all the available high-density data and satellite data into the initial field of typhoon to reconstruct the inner structure of typhoon, and the 48 hours track forecasting error was reduced from $400 \mathrm{~km}$ in the control experiment to $150 \mathrm{~km}$ in the assimilating experiment. Wang et al. [17] effectively improved the intensity forecasting and track forecasting of typhoon, by simultaneously assimilating the large-scale background fields data, bogus data, cloud-derived wind data, satellite inverse data, high-resolution infrared radiation sounder data (HIRS), and so forth. into the numerical forecasting model.

Zhang et al. [18] adopted 3-dimensional variational assimilation (3DVAR) technique to assimilate AMSU brightness temperature data into numerical model in the study of the typhoon structure and its evolution in different stages on Northwest Pacific. The numerical results showed that the direct assimilation of AMSU brightness temperature data could accurately describe the 3-dimensional structure and evolution of typhoon on Northwest Pacific, which is difficult to achieve with conventional observation data. The study shows that the AMSU data provide improvement to track forecasts of tropical cyclone [19].

Though BDA method and AMSU-A data assimilation method have been used many times in numerical simulating of hurricane, there is few research work combining the two methods by 4DVAR technique in typhoon simulating. Selecting typhoon "Aere" (No. 0418) as the case, this paper combines BDA method with AMSU-A data assimilation method to conduct assimilating experiments and numerical simulating experiments to reconstruct mesoscale information of typhoon, and also studies the intensity forecasting and track forecasting. The numerical models, the observation data, and the objective function are briefly introduced in Section 2. Experiment design of numerical scheme and the corresponding numerical results are shown in Section 3. Section 4 provides conclusions and discussion.

\section{Numerical Models, Observation Data, and Objective Function}

2.1. Numerical Models. The MM5V3 model, the RTTOV7 model, and their adjoint models are adopted in this paper. The MM5 model is a mesoscale numerical forecasting model which is developed jointly by National Center for Atmospheric Research (NCAR) and Pennsylvania University (PSU). In this paper, the central point of the numerical simulating domain is $\left(124.5^{\circ} \mathrm{E}, 22.5^{\circ} \mathrm{N}\right)$. The assimilating experiments are conducted on single-nest domain with $54 \mathrm{~km}$ horizontal resolution and the horizontal grid number is $75 \times 91$. The simulating experiments are conducted on double-nest domain. The horizontal resolutions are, respectively, $54 \mathrm{~km}, 18 \mathrm{~km}$, and horizontal grids are, respectively, 75 $\times 91,121 \times 130$. The $\sigma$-coordinate is used in the numerical model and there are totally 23 levels in the vertical direction. Parameterization schemes used in simulation are as the followings: Grell cumulus parameterization scheme [20], MRF PBL scheme [21], relaxation inflow/outflow boundary condition, and so forth.

The RTTOV radiative transfer model is to compute very rapid calculations of top of atmosphere radiances for a range of space-borne infrared and microwave radiometers viewing the Earth's atmosphere and surface. The original basis for the RTTOV fast computation of transmittances is described by Eyre and Woolf [22]. The development of RTTOV-7 has been carried out as part of collaboration between the Met Office (UK), Météo France, and ECMWF in the framework of the EUMETSAT-funded NWP Satellite Application Facility and also other EUMETSAT sponsored activities. The RTTOV7 model describes the complicated nonlinear relationship between the satellite radiation brightness temperature and the model variables. Various physical fields such as temperature profile and water vapor profile required by the RTTOV-7 model are provided by the MM5V3 model. In the RTTOV-7 model, the atmosphere is divided into 43 layers from $0.1 \mathrm{hPa}$ to $1013 \mathrm{hPa}$ and the channel transmissivity is calculated layer by layer according to the actual vertical structure of atmosphere so that the radiant brightness temperature of every channel can be finally synthesized. Different from 
the traditional radiant model, the optics thickness $d$ in the RTTOVS-7 model can be expressed as

$$
d_{i, j}=d_{i, j-1}+Y_{j} \sum_{k=1}^{K} a_{i, j, k} X_{k, j},
$$

where $Y_{j}$ and $X_{k, j}$ are factors related with the actual atmospheric condition, $a_{i, j, k}$ is the forecast factor obtained by the accurate transmissivity model, $i$ is the serial number of channels, $j$ is the serial number of different levels, and $k$ is the serial number of forecast factors.

2.2. Observation Data. The National Centers for Environmental Prediction (NCEP) Global Forecast System (GFS) final (FNL) girded analysis datasets, with horizontal resolution of $1^{\circ} \times 1^{\circ}$, are used to produce the background fields of model.

In BDA method, the bogus data, including the sea level pressure and wind fields, are calculated based on observational typhoon information by the following formulas:

$$
\begin{gathered}
P_{0}(r)=P_{c}+\Delta P\left\{1-\left[1+\frac{1}{2}\left(\frac{r}{R}\right)^{2}\right]^{-1 / 2}\right\}, \\
V_{0}(r)=\left(\frac{r}{\rho} \frac{\partial P_{0}}{\partial r}+\frac{f^{2} r^{2}}{4}\right)^{1 / 2}-\frac{r|f|}{2},
\end{gathered}
$$

where $P_{c}$ is the sea level pressure of typhoon center; $\Delta P$ is a parameter related with the sea level pressure gradient and is determined by the maximum wind speed of typhoon; $R$ is the radius of the maximum pressure gradient, usually that of the maximum wind speed; $r$ is the radius of vortex; $\rho$ is the air density. The bogus typhoon is regarded as quasi-stationary in the first 32 minutes. The time window of assimilating bogus data is set to 32 minutes, and the same "observed data" is read every 4 minutes [8].

The NOAA-16 AMSU-A data provided by National Aeronautics and Space Administration (NASA) are used in this paper. There are totally 15 detective channels of AMSUA data, which has been interpolated to match the horizontal resolution of HIRS data. Finally, the resolution of subsatellite point is $17.4 \mathrm{~km}$. In every scanning line there are 56 detective pixels which are axisymmetric along the satellite orbit. In this paper, the presence of precipitation cloud has already been checked before the performance of 4DVAR process. Despite that AMSU has the ability to penetrate the cloud layer to detect atmospheric temperature and humidity, while the water droplets and ice crystals in precipitation cloud are larger than the radiance wavelength, so the resulting scatter can weaken the signal below the cloud layer. Since it influences the detection, the presence of precipitation cloud must be checked. The test formula of the precipitation probability $P$ (unit: \%) of the ATOVS is expressed as follows [18]:

$$
\begin{gathered}
P=\frac{1}{1+e^{-f}}, \\
f=10.5+0.184 T B_{1}-0.221 T B_{15},
\end{gathered}
$$

where $T B_{1}$ and $T B_{15}$ are the observation brightness temperature in channel 1 and channel 15 , respectively. When $P \geqq$ 70 , the radiance brightness temperature must be rejected. During the assimilation procedure of AMSU-A data, quality control method and channel choosing method are both taken into consideration. Two quality control techniques are employed: extreme value check and a check for departures between the simulated observation value and the actual observation value. The channel is selected according to the peak energy contribution level of the sounder channel and the influence of the sounding objective on the retrieval results of temperature and water vapor. In fact, the 4 channels from 6 to 9 are adopted in this paper.

2.3. Objective Function. When the bogus data and AMSU-A data are assimilated into model at the same time, the total objective function $J$ can be defined as

$$
J=J_{1}+J_{2}+J_{3},
$$

where $J_{1}$ is the deviation between the model control variable $X$ and the background variable $X_{B}, J_{2}$ is that between the model control variable and the bogus data, and $J_{3}$ is that between the simulated AMSU-A brightness temperature and the actual satellite observation data. Their general form can be expressed as follows:

$$
\begin{gathered}
J=\frac{1}{2} \sum_{i}\left(X-X_{B}\right)^{T} B^{-1}\left(X-X_{B}\right), \\
J_{3}=\frac{1}{2} \sum_{t} \sum_{i}\left\{\left[P(r)-P_{0}(r)\right]^{T} W_{P}\left[P(r)-P_{0}(r)\right]\right. \\
\left.\quad+\left[V(r)-V_{0}(r)\right]^{T} W_{V}\left[V(r)-V_{0}(r)\right]\right\}, \\
J_{3}=\frac{1}{2} \sum_{t} \sum_{i}\left[H_{B T A}(X)-B T A\right]^{T} W_{B T A}\left[H_{B T A}(X)-B T A\right],
\end{gathered}
$$

where the superscript " $T$ " denotes transpose, $B$ is the error covariance matrix of background fields, $P(r)$ and $V(r)$ are, respectively, sea level pressure and sea level wind of model atmosphere, $W_{P}$ and $W_{V}$ are the corresponding weighting coefficients, " $\sum$ " denotes the sum, the subscript sign " $t$ " denotes different observing times, the subscript sign " $i$ " denotes different spatial observing positions at the same time, $H_{B T A}$ denotes the observed operator of calculating the simulated brightness temperature with model atmospheric variables, $B T A$ denotes the actual AMSU-A observation data, and $W_{B T A}$ denotes the weighting coefficient of detective channels. We take $W_{p}=1.61 / \mathrm{hPa}^{2}$ and $W_{v}=0.185 \mathrm{~S}^{2} / \mathrm{m}^{2}$ in all experiments, just like Xiao et al. [8]. $W_{B T A}$ is taken according to the following principle: the weighting coefficients of different channels should have the same contribution to the total cost function. In this paper, they are, respectively, 10.41, 9.24, 12.54, and 11.90.

\section{Numerical Experiments}

3.1. Scheme Designs. Typhoon "Aere" (no. 0418) was generated over the sea at about $1400 \mathrm{~km}$ away from the east 
TABLE 1: The schemes of numerical simulation and assimilating experiment.

\begin{tabular}{ll}
\hline Scheme & Initial fields of model \\
\hline Control experiment (Scheme 1) & Provided by NCEP data \\
Bogus data assimilation experiment (Scheme 2) & Provided by BDA method \\
Total data assimilation experiment (Scheme 3) & Provided by BDA method combined with AMSU-A data assimilation method \\
\hline
\end{tabular}

of Luzon, Philippines on August 20, 2004, and then it moved west-northward under the lead of the subtropical high, with maximum wind speed of up to $43 \mathrm{~m} / \mathrm{s}$. "Aere" swept the adjacent waters to northern Taiwan on dawn of August 25 and in the same afternoon it landed on Fujian province of China with wind speed of $35 \mathrm{~m} / \mathrm{s}$. After landing it gradually downgraded to a tropical depression. This paper focuses on typhoon intensity forecasting, typhoon track changing, landing and vapor variation. Numerical simulation is performed for 72 hours with the initial time set at 12:00 on August 23. The satellite profiles at 17:45 on August 23 are assimilated within 345 minutes, with the assimilating window set to 360 minutes.

The schemes of numerical simulation and assimilating experiments are shown in Table 1, including a control experiment (Scheme 1) and two assimilating experiments (Schemes 2 and 3).

\subsection{Numerical Results}

3.2.1. The Initial Fields. The initial fields of three schemes are shown in Figure 1, in which the left panels are sea level pressure fields (units: $\mathrm{hPa}$ ) and the right are temperature fields at $500 \mathrm{hPa}$ (units: ${ }^{\circ} \mathrm{C}$ ).

Figure 1(a) shows that the isobars on sea level at initial time provided by NCEP data are very sparse. The minimum central sea level pressure is $988 \mathrm{hPa}$ while the actual observed value is $975 \mathrm{hPa}$. Figure 1(c) indicates that the intensity of initial typhoon is effectively enhanced, with central sea level pressure of $975 \mathrm{hPa}$ after the bogus data is assimilated, which is in accordance with the observed value. Figure 1(e) indicates that the intensity of initial typhoon in Scheme 3 is also $975 \mathrm{hPa}$ when BDA method and AMSU-A data assimilation method are combined. In fact, when only the AMSU-A data is assimilated, the intensity of initial typhoon would not obviously be improved. This implies that BDA method is more effective than AMSU-A data assimilation method in improving the intensity of initial typhoon.

Figure 1(b) shows that the "warm core" structure of initial temperature fields in NCEP data is not obvious and the central temperature of initial typhoon is about $0^{\circ} \mathrm{C}$. Therefore, the weak "warm core" will greatly influence the forecasting intensity and forecasting track. Figures 1(d) and 1 (f) show that there are obvious "warm core" structures both in Schemes 2 and 3, in which the maximum central temperature is $3.5^{\circ}$ and $3.3^{\circ}$, respectively. It denotes that data assimilation is effective in improving temperature fields of initial typhoon, especially in Scheme 3, when AMSUA data are assimilated, more mesoscale information are added into initial temperature fields. In the two assimilating schemes, although temperature data is not directly assimilated, temperature fields can still be adjusted correspondingly under the constraints of model dynamics whenever the other observation data are assimilated; it is a significant advantage of 4DVAR technique.

The vorticity fields of typhoon at initial time in the three schemes are shown in Figure 2. The left panels denote the horizontal vorticity patterns on $300 \mathrm{hPa}$ at initial time and the right ones denote the vertical vorticity patterns at initial time (vertical sections are given cross the center of typhoon along the zonal direction, where arrows denote the synthetic effect of horizontal velocity $\mathrm{v}$ and vertical velocity $\left.w^{*} 200\right)$. Comparing Figure 2(a) with Figure 2(c), it can be found that the effect of the BDA method is not remarkable in improving vorticity fields at upper troposphere. Figure 2(e) indicates that when AMSU-A data is assimilated the vorticity fields of typhoon at initial time change obviously and many mesoscale structures are reconstructed. Comparison between Figures 2(b) and 2(d) shows that although the BDA method can enhance the inner vertical circulation of the initial typhoon, it has little influence on the typhoon circulation at upper troposphere. Figure 2(f) shows that when BDA method is combined with AMSU-A data assimilation method, a lot of meso- and microscale information are introduced into the vertical vorticity fields, which proves the positive effect of assimilating AMSU-A brightness temperature data on typhoon numerical forecasting. Though the BDA method is not very effective in improving the initial typhoon circulation fields at high level, it is more effective in decreasing sea level pressure of initial typhoon. And the direct assimilation of AMSU-A data is not effective in improving the intensity of initial typhoon, but it does well in improving the circulation structure of initial typhoon at upper troposphere. Therefore, in order to improve typhoon numerical forecasting, combining BDA method and AMSUA data assimilation method would be beneficial to optimize the initial fields separately from low level and high level. In this way, not only the intensity of initial typhoon can be improved, but also the mesoscale structure of initial typhoon can be reconstructed.

It is well known that there is no cloud water in the initial fields of numerical model, just like in Scheme 1. But the cloud water of the optimal initial fields will increase during the 4DVAR iterative process by assimilating observation data. That is to say, the cloud water is formed by MM5 adjoint model under the constraints of model dynamics. Figure 3 shows the cloud water content (units: $\mathrm{kg} / \mathrm{kg}$ ) of the optimal initial fields on $400 \mathrm{hPa}$ in Schemes 2 and 3. In Scheme 2, the circular cloud water is formed in the optimal initial fields due to assimilating the bogus data. While in Scheme 3, a 


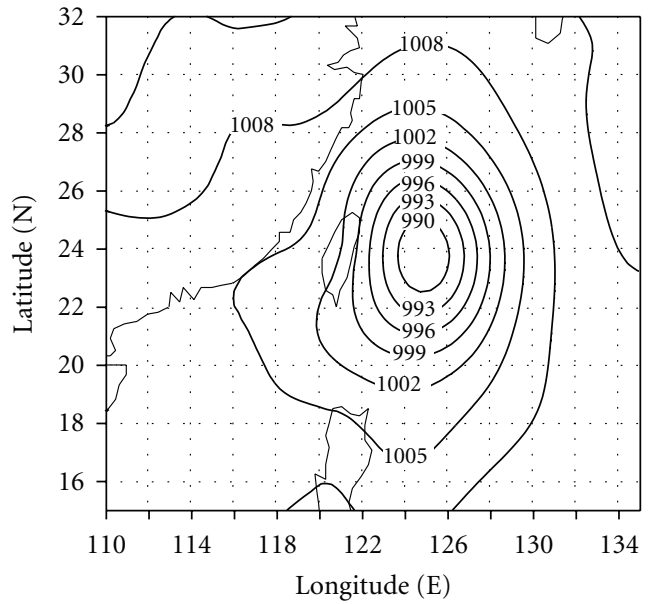

(a)

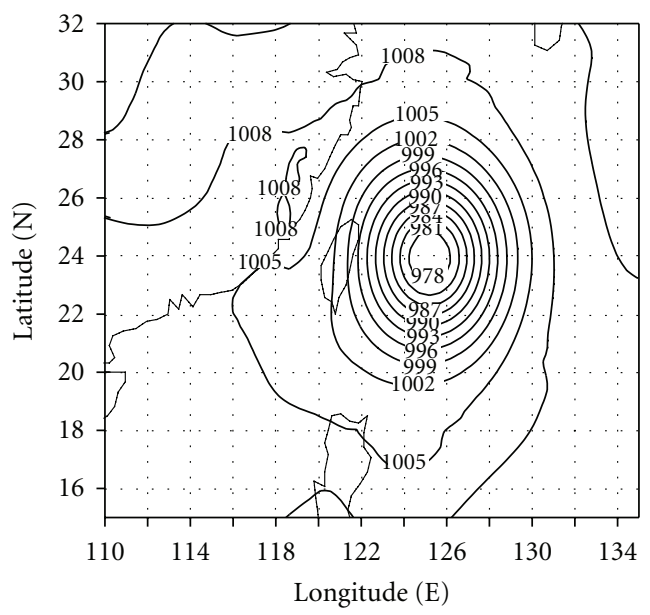

(c)

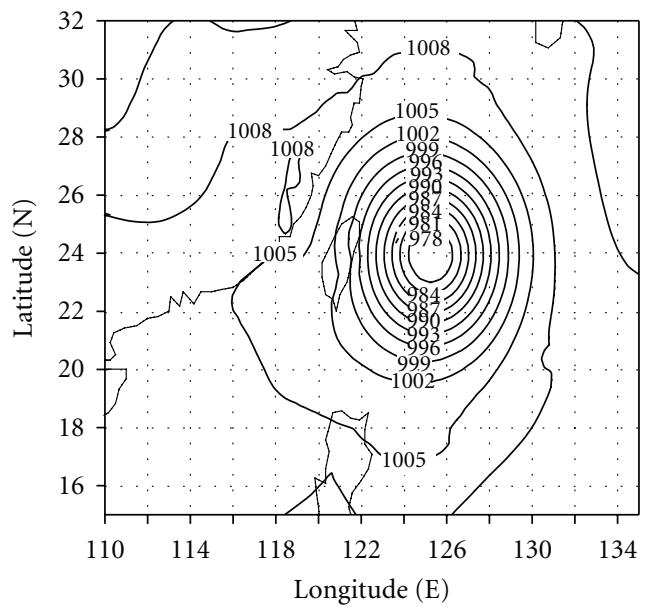

(e)

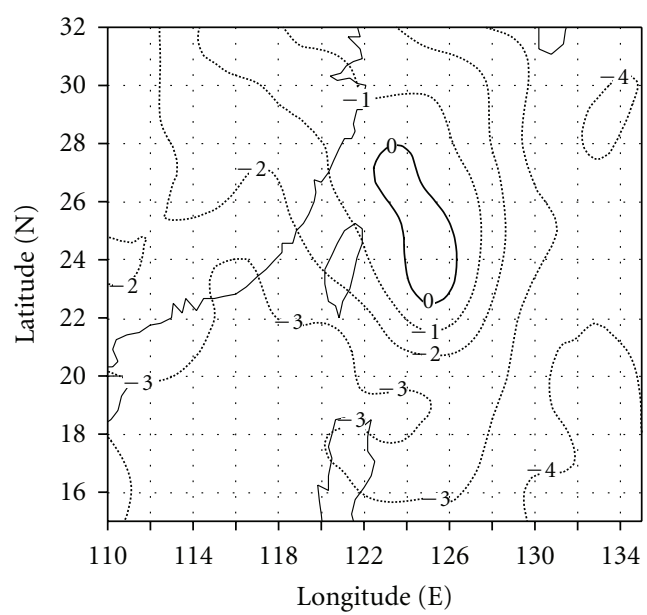

(b)

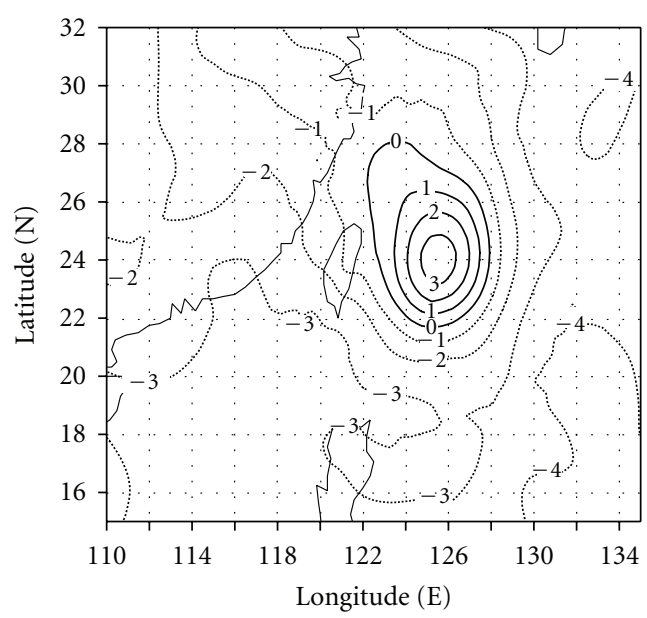

(d)

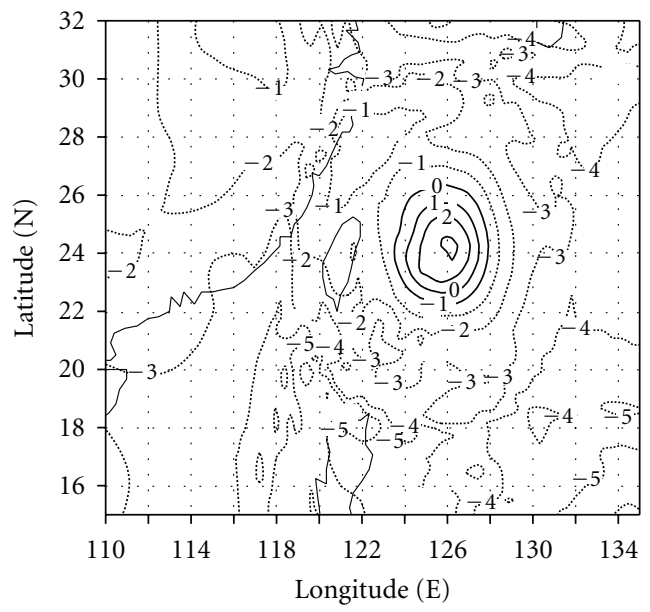

(f)

Figure 1: The initial fields of sea level pressure ((a) Scheme 1, (c) Scheme 2, (e) Scheme 3; hPa) and temperature ((b) Scheme 1, (d) Scheme 2, (f) Scheme $3 ;{ }^{\circ} \mathrm{C}$ ) simulated by various experiments at $500 \mathrm{hPa}$. 


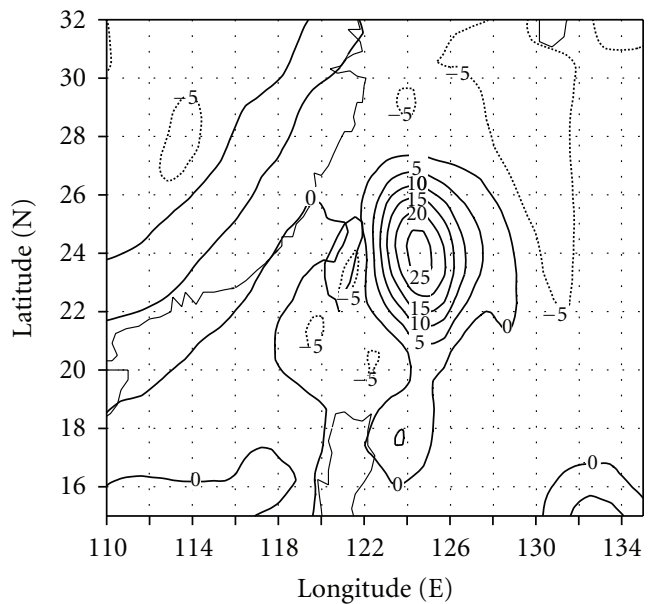

(a)

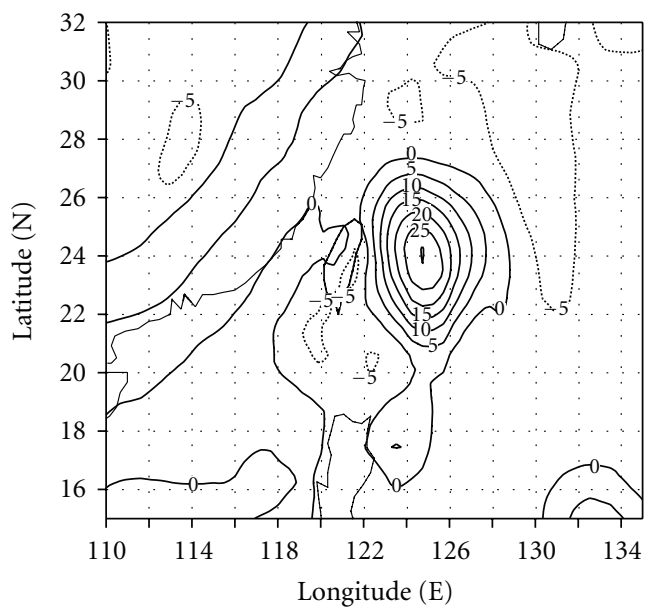

(c)

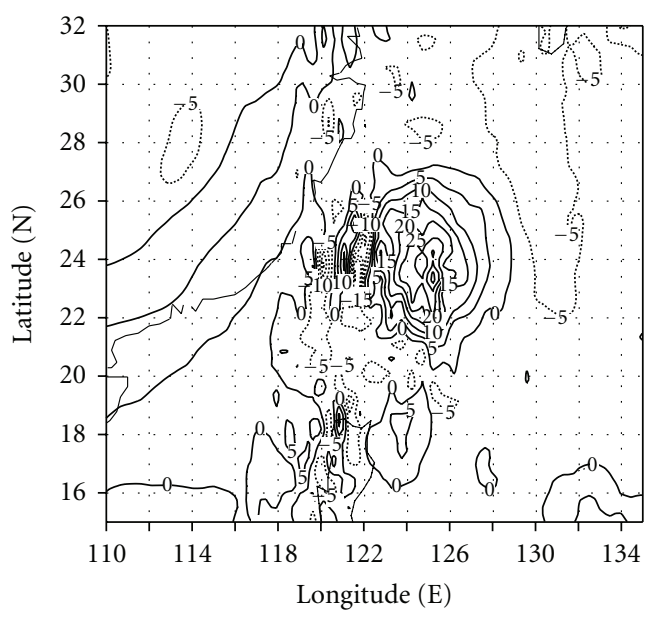

(e)

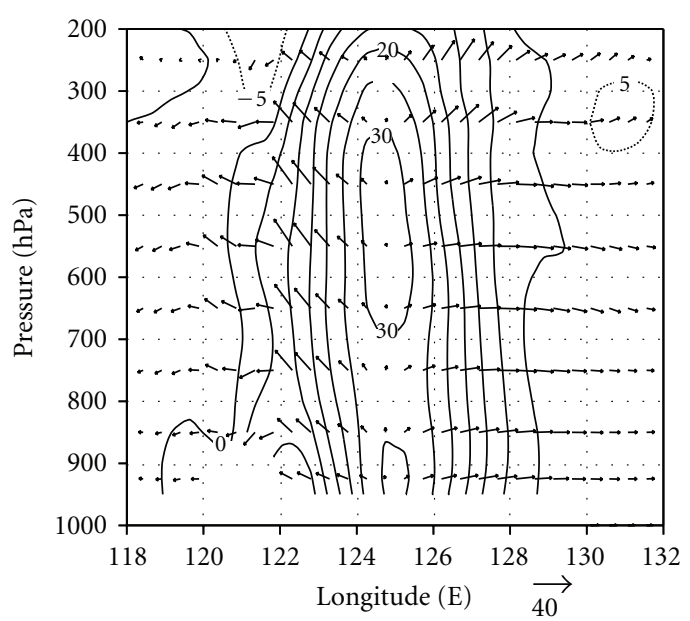

(b)

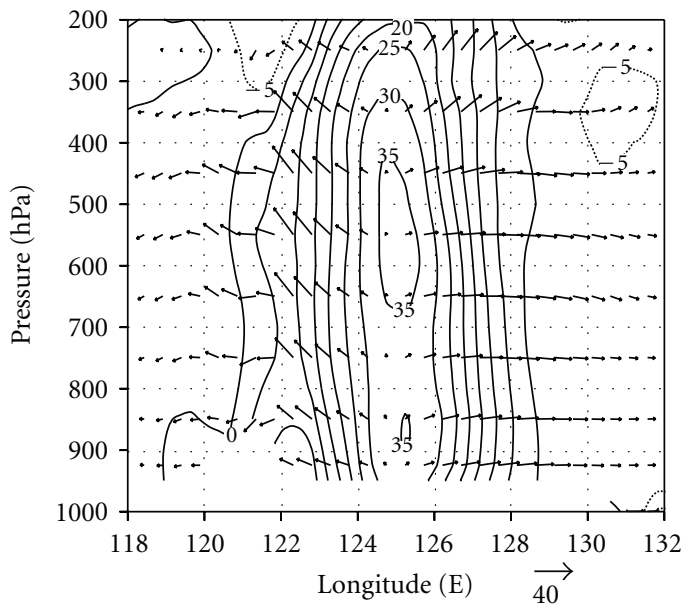

(d)

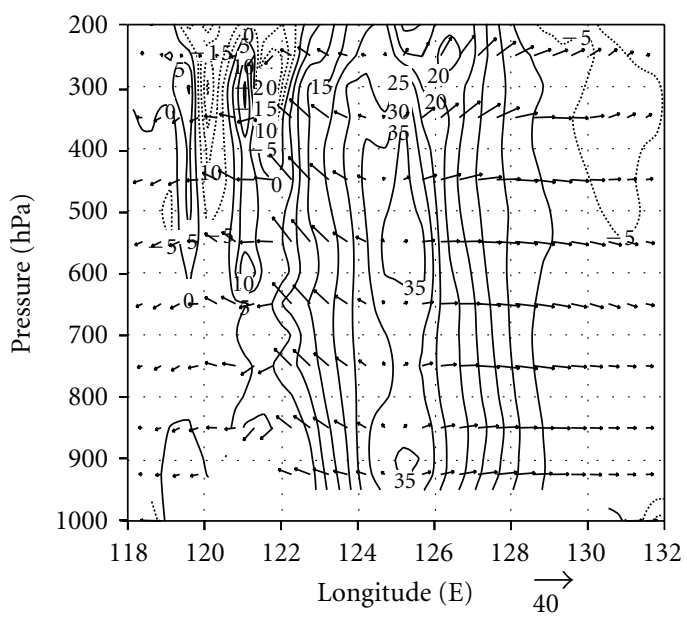

(f)

FIgure 2: The initial horizontal vorticity fields $(* 1 . e+5)$ on $300 \mathrm{hPa}\left((\mathrm{a})\right.$ Scheme 1, (c) Scheme 2, (e) Scheme 3; $\left.\mathrm{s}^{-1}\right)$ and vertical vorticity fields and wind fields $\left(\mathrm{w}^{*} 200\right)$ cross the center of typhoon along the zonal direction ((b) Scheme 1, (d) Scheme 2, (f) Scheme 3; ${ }^{-1}$ ) simulated by various experiments. 


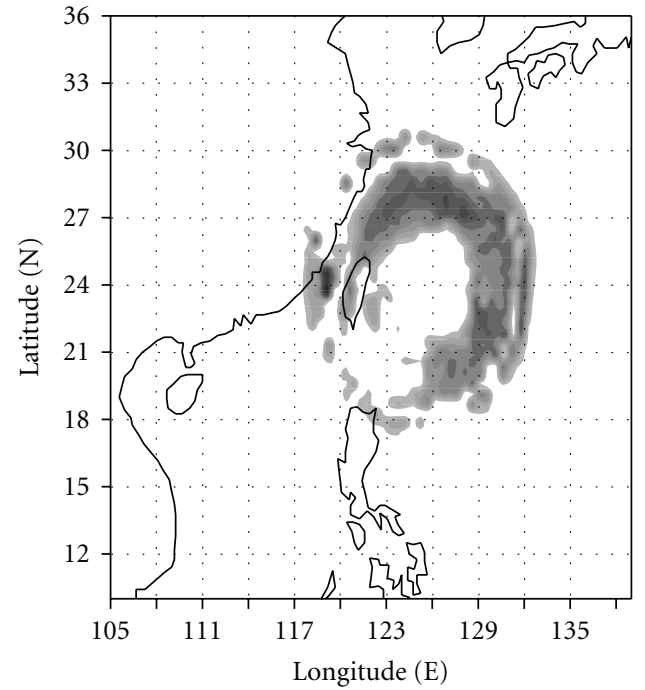

(a)

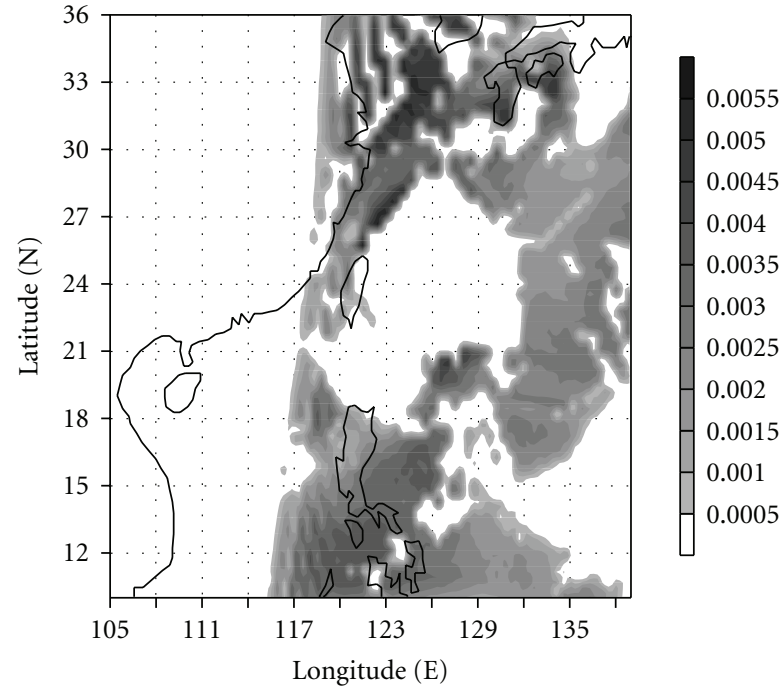

(b)

Figure 3: Cloud water of optimal initial fields in different Schemes on $400 \mathrm{hPa}$ ((a) Scheme 2, (b) Scheme 3; kg/kg).

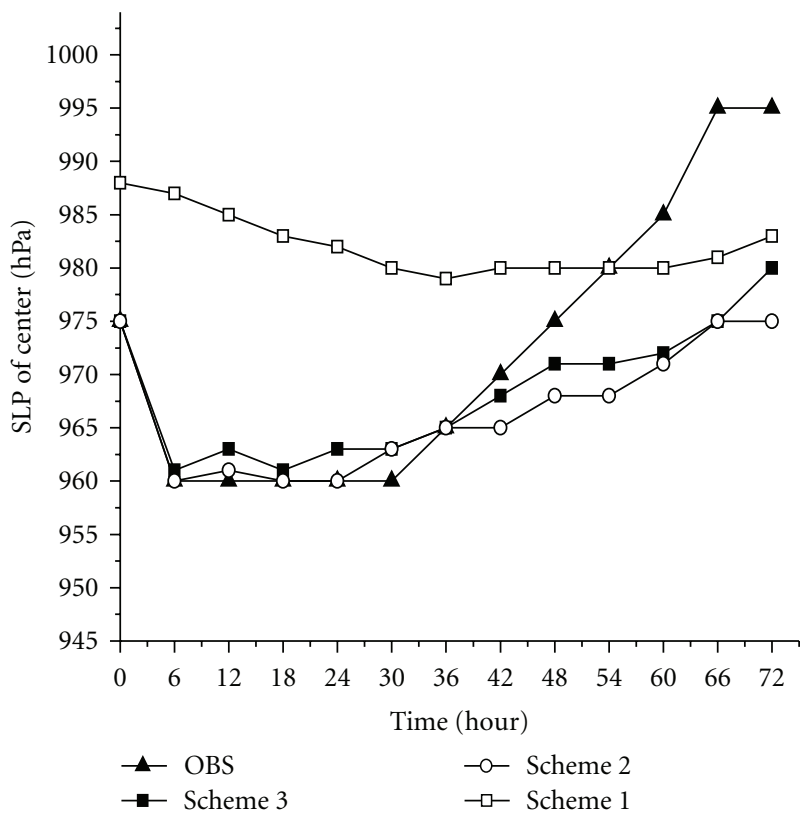

FIGURE 4: Simulated SLP of the center of typhoon during 72 hours in the three schemes Triangle point line: Observed data (OBS); Hollow square point line: Scheme 1; Hollow dot point line: Scheme 2; Solid square point line: Scheme 3.

reasonable cloud water pattern is formed due to assimilating the AMSU-A data. The coverage of satellite scanning can also be clearly shown in Figure 3(b), especially the location of actual typhoon, which is denoted by blank section in the center of figure.

3.2.2. Intensity of Simulated Typhoon during 72 Hours in the Three Schemes. The varying curves of SLP (sea level pressure) of simulated typhoon during 72 hours in the three schemes are shown in Figure 4. Figure 4 shows that the typhoon intensity in Scheme 1 varies gently during the whole period, which is quite different from the trend of observed data, with the bias of $13 \mathrm{hPa}$ at the initial time, the maximum bias of $27 \mathrm{hPa}$ and the mean bias of $15 \mathrm{hPa}$. In Schemes 2 and 3, the advantage of BDA method is brought into fullest play. In both schemes, the central intensity of initial typhoon is improved greatly, and the central intensity of initial typhoon is in complete accordance with observation data $(975 \mathrm{hPa})$. Furthermore, the simulated SLP during 72 hours is also improved greatly and its general varying trend is in accordance with OBS, with the mean bias of $6.3 \mathrm{hPa}$ in Scheme 2 and $5.6 \mathrm{hPa}$ in Scheme 3. The best one is the former 48 hours, during which the maintaining stage of typhoon is well simulated, with the mean biases of less than $3 \mathrm{hPa}$. The intensity changes are closely related with landfall. If the landfall time is not well predicted, it is difficult to have a good intensity prediction of landfall typhoons. The typhoons in Schemes 1 and 2 do not land at all, while it is delayed for about 12 hours in Scheme 3. So for the last 24 hours, the observed typhoon is weakened rapidly after landfall, while the simulated typhoon intensities in three schemes are varying slowly. However, Scheme 3 is better than Scheme 2 in the last 24 hours, and both Schemes 2 and 3 are far better than Scheme 1 when the whole period is concerned. Besides, because the assimilating of AMSU-A data is added into Scheme 3, the mesoscale information of initial fields is further increased and the surrounding fields of initial typhoon can be well simulated. Since the initial fields are further adjusted with AMSU-A data assimilation method, the simulating results of Scheme 3 are better than those of Scheme 2.

3.2.3. Track of Simulated Typhoon during 72 Hours in the Three Schemes. Comparison of typhoon track in the three schemes with observed typhoon track during the simulated 


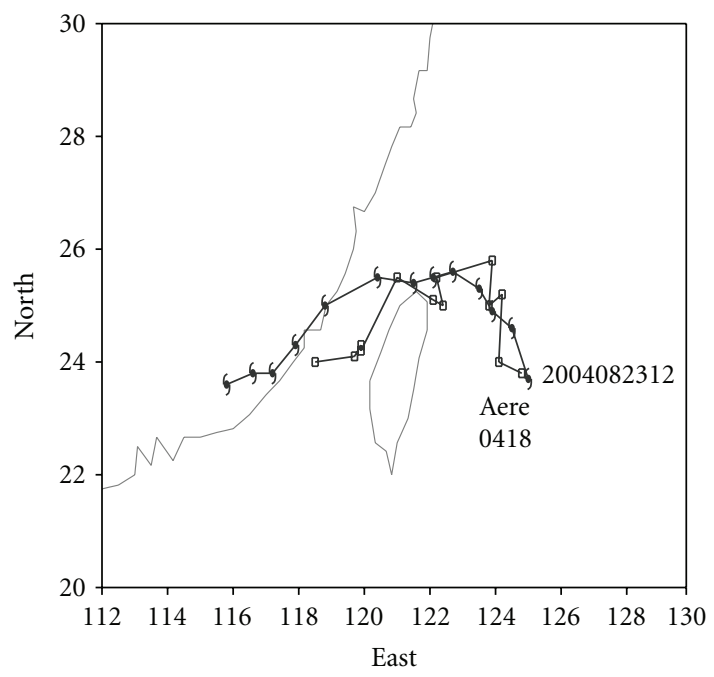

(a)

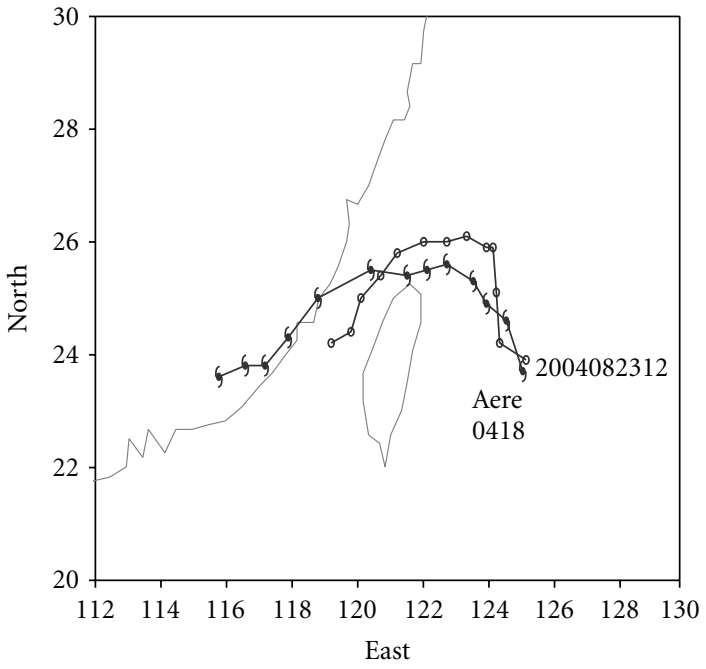

(b)

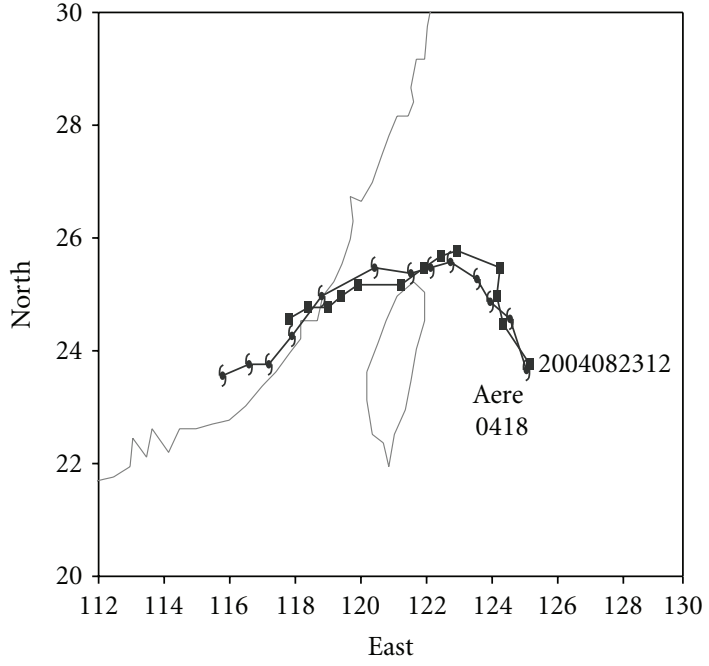

(c)

FIGURE 5: The varying curves of simulated typhoon track during 72 hours in the three schemes ((a) Scheme 1, (b) Scheme 2, (c) Scheme 3 ) Typhoon marker point line: Observed data; Hollow square point line: Scheme 1; Hollow dot point line: Scheme 2; Solid square point line: Scheme 3.

72 hours is shown in Figure 5. The figure shows that the observed typhoon firstly moves to the west-north and then sweeps over the adjacent waters to northern Taiwan, and finally lands on Fujian province of China. Figure 5(a) shows that the simulated track of typhoon in Scheme 1 is greatly different from the observed track; the simulated typhoon moves with irregular speed. Figure 5(b) shows that the simulated track of typhoon in Scheme 2 is close to the observed data, but the typhoon moves slowly and does not land on Fujian province of China at last. Figure 5(c) shows that the simulated track of typhoon in Scheme 3 is the best one, in which the whole moving trend of typhoon is closest to the observed data and the simulated typhoon finally lands on Fujian province of China, with landing location in accordance with the observed data, although its moving speed is still smaller than that of the observed data.
The bias curves of simulated typhoon track in three schemes from the observed track during 72 hours are shown in Figure 6. From Figure 6 it can be found that the location of typhoon at initial time in Scheme 3 is the closest to the observed typhoon location, with $15 \mathrm{~km}$ distance away from each other. In the following period, the biases of Scheme 3 are also smaller than those of other schemes, with $112 \mathrm{~km}$ in Scheme 3 while $228 \mathrm{~km}$ in Scheme 1 and $256 \mathrm{~km}$ in Scheme 2 in 48 hours; $231 \mathrm{~km}$ in Scheme 3 while $278 \mathrm{~km}$ in Scheme 1 and $351 \mathrm{~km}$ in Scheme 2 in 72 hours. Therefore, it can be concluded that although the BDA method is effective in improving typhoon intensity in the simulation, it could not much effectively improve typhoon track. The AMSUA data assimilation method has no significant effect on improving typhoon intensity but it can greatly reconstruct mesoscale information of initial fields and greatly improve 
TABLE 2: The mean forecast biases of 72 hours for different typhoons.

\begin{tabular}{lcccccc}
\hline Typhoon & & Intensity biases $(\mathrm{hPa})$ & \multicolumn{3}{c}{ Track biases (km) } \\
& Scheme 1 & Scheme 2 & Scheme 3 & Scheme 1 & Scheme 2 & Scheme 3 \\
\hline Aere (0418) & 15 & 6.3 & 5.6 & 146 & 179 & 97 \\
Meari (0422) & 39 & 5 & 4 & 220 & 195 \\
Dianmu (0406) & 54 & 8.7 & 8.6 & 191 & 136 \\
\hline
\end{tabular}

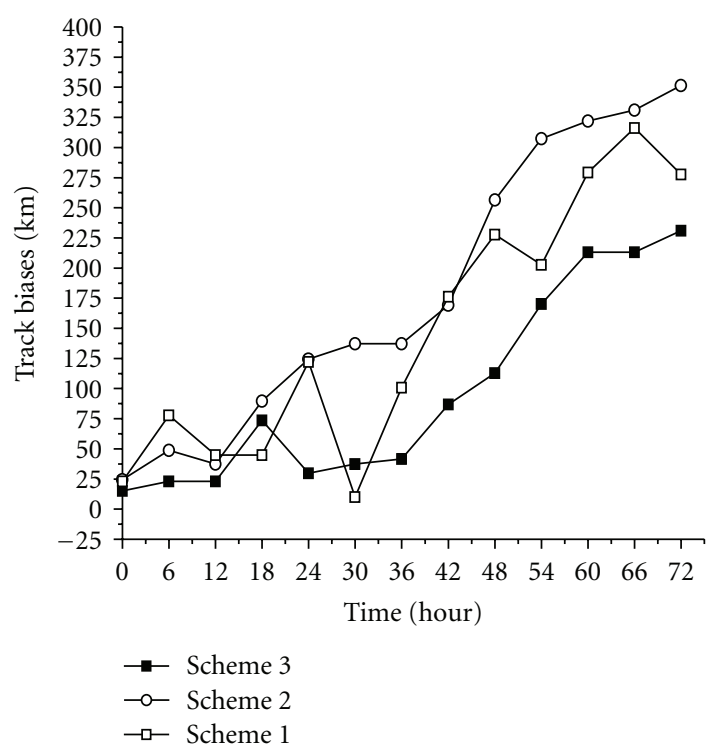

Figure 6: Biases of simulated typhoon track during 72 hours in the three schemes (Hollow square point line, Scheme 1; Hollow dot point line, Scheme 2; Solid square point line, Scheme 3).

typhoon track simulation. Besides, the intensity changes are closely related with landfall. If the landfall time is not well predicted, it is difficult to have a good intensity prediction of landfall typhoons. So for the last 24 hours, the simulated typhoon intensity in Scheme 3 is closer to the observed one than in Scheme 2. It further proves that typhoon intensity simulation and typhoon track simulation can be improved simultaneously by combining BDA method and AMSU-A data assimilation method. The typhoons in Schemes 1 and 2 do not land at all, while in Scheme 3 it is delayed for about 12 hours.

By comparing Figure 1(d) (Scheme 2) with Figure 1(f) (Scheme 3), and by comparing Figure 2(d) (Scheme 2) with Figure 2(f) (Scheme 3), we can find that many mesoscale/microscale systems are introduced into initial vorticity fields at high level of atmosphere when AMSU-A data is assimilated, and the AMSU-A data helps to improve the circulation of typhoon at high level. In our cases, the AMSU-A data assimilation is helpful to improve typhoon track forecast. In order to reveal the reasons, we also study the vertical structure of temperature fields and geopotential height fields. It is shown that temperature difference and geopotential height difference of optimal fields (Scheme 3 minus Scheme 2) are both located at high level, especially

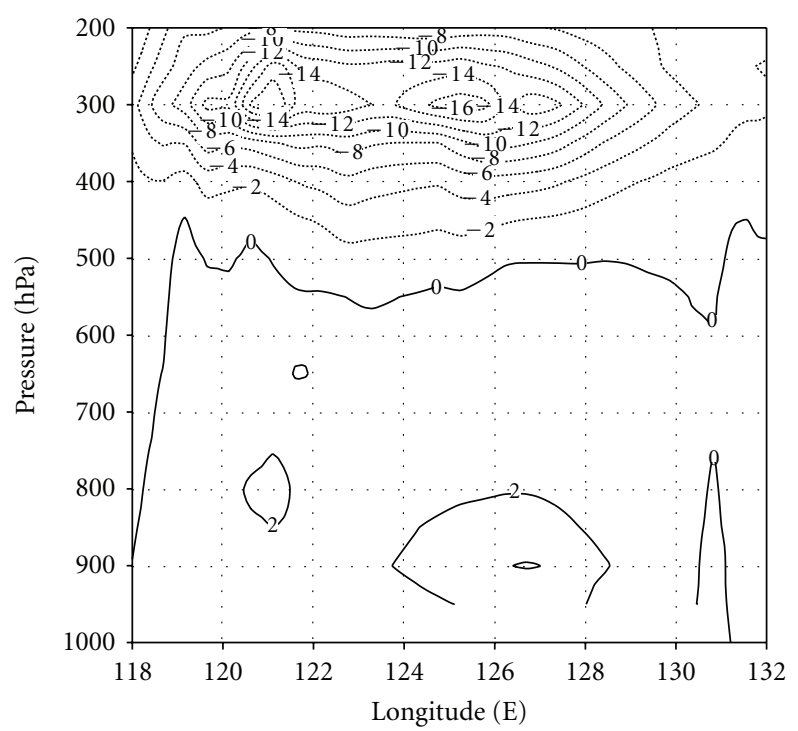

(a)

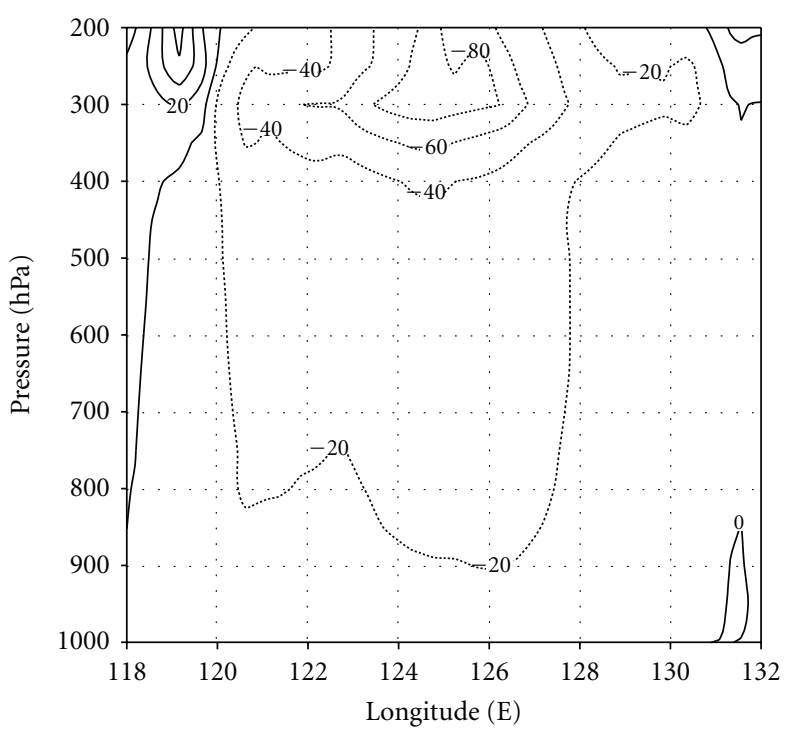

(b)

Figure 7: The vertical sections of temperature difference $\left((\mathrm{a}) ;{ }^{\circ} \mathrm{C}\right)$ and geopotential height difference cross the center of typhoon along the zonal direction $((\mathrm{b}) ; \mathrm{m})$ between Schemes 3 and 2 (Scheme 3 minus Scheme 2) in optimal initial fields.

at $300 \mathrm{hPa}$ (shown as Figure 7). The results illustrate that AMSU-A data assimilation can be helpful to improve the high-level fields. In this paper, channels $6-9$ of AMSU-A 
data are selected in assimilation experiments. Because their peak energy contribution levels are mainly located at upper troposphere, the observed information of AMSU-A data mainly concentrates at upper troposphere and AMSU-A data assimilation can have a positive effect on reconstructing the mesoscale information at upper troposphere. This is the reason that though the direct assimilation of AMSU-A data is not effective in improving the intensity of initial typhoon, it does well in improving the circulation structure of initial typhoon at upper troposphere.

Some assimilating experiments and simulating experiments on other typhoons are also performed by use of BDA method combined with AMSU-A data assimilation method, and the same numerical conclusions can also be achieved. The results are shown in Table 2 .

\section{Conclusions}

With BDA method combined with AMSU-A data assimilation method, the assimilating experiments and simulating experiments about some typhoons are carried out in this paper by using the MM5V3 model, the RTTOV-7 model and their adjoint models. The numerical results show the following.

(1) If the initial fields are directly provided by NCEP data, the isobars on sea level at initial time are very sparse and the "warm core" structure of initial temperature fields is not obvious. Compared with the real observations, there are big biases both in intensity simulation and track simulation.

(2) After being adjusted with the BDA method, the initial fields can effectively describe the initial circulation structure of typhoon. The BDA method can enhance initial typhoon intensity and improve initial typhoon thermal structure such as "warm core" structure. Though this method is effective in improving typhoon intensity simulating, it has no significant effect on improving typhoon track.

(3) Combining BDA method and AMSU-A data assimilation method can improve not only typhoon intensity simulation but also typhoon track simulation. By use of 4DVAR technique, the circulation structure of analytical typhoon is greatly improved and the "warm core" structure is also enhanced. "Cloud water" arises in optimal initial fields of typhoon after observed data are assimilated. The running status of numerical model is changed into "warm start" from "cold start", which is more reasonable. So combining BDA method and AMSU-A data assimilation method effectively may be an important way to increase the accuracy of typhoon numerical forecasting.

Though some interesting results have been obtained in this paper, further more experiments on other typhoons should be performed to confirm them. And AMSU-B data and HIRS data will be assimilated in further research work to improve the initial fields of typhoon.

\section{Acknowledgments}

This paper is jointly supported by the National Natural Science Foundation of China (no. 40405019, no. 40805046, and no. 10871099), the Commonweal Trade (Meteorology) Program (no. GYHY(QX)2007-6-15 and no. GYHY200906009), and the National Basic Research Program of China (973 Program) (no. 2009CB421500).

\section{References}

[1] T. Iwasaki, H. Nakano, and H. Sugi, "The performance of a typhoon track prediction model," Journal of the Meteorological Society of Japan, vol. 65, pp. 555-570, 1987.

[2] M. B. Mathur, "The National Meteorological Center's quasilagrangian model for hurricane prediction," Monthly Weather Review, vol. 119, no. 6, pp. 1419-1447, 1991.

[3] Y. Kurihara, M. A. Bender, and R. J. Ross, "An initialization scheme of hurricane models by vortex specification," Monthly Weather Review, vol. 121, no. 7, pp. 2030-2045, 1993.

[4] J. M. Lewis and J. C. Derber, "The use of adjoint equation to solve a variational adjustment problem with advective constraints," Tellus, vol. 37A, pp. 309-322, 1985.

[5] F.-X. Le Dimet and O. Talagrand, "Variational algorithms for analysis and assimilation of meteorological observations: theoretical aspects," Tellus, vol. 38A, no. 2, pp. 97-110, 1986.

[6] P. Courtier and O. Talagrand, "Variational assimilation of meteorological observations with the adjoint vorticity equation. Part I: theory," Quarterly Journal of the Royal Meteorological Society, vol. 113, no. 478, pp. 1311-1328, 1987.

[7] X. Zou and Q. Xiao, "Studies on the initialization and simulation of a mature hurricane using a variational bogus data assimilation scheme," Journal of the Atmospheric Sciences, vol. 57, no. 6, pp. 836-860, 2000.

[8] Q. Xiao, X. Zou, and B. Wang, "Initialization and simulation of a landfalling hurricane using a variational bogus data assimilation scheme," Monthly Weather Review, vol. 128, no. 7, pp. 2252-2269, 2000.

[9] Z.-X. Pu and S. A. Braun, "Evaluation of bogus vortex techniques with four-dimensional variational data assimilation," Monthly Weather Review, vol. 129, no. 8, pp. 2023-2039, 2001.

[10] F. Weng, T. Zhu, and B. Yan, "Satellite data assimilation in numerical weather prediction models. Part II: uses of rainaffected radiances from microwave observations for hurricane vortex analysis," Journal of the Atmospheric Sciences, vol. 64, no. 11, pp. 3910-3925, 2007.

[11] Q. Xiao, L. Chen, and X. Zhang, "Evaluations of BDA scheme using the advanced research WRF (ARW) model," Journal of Applied Meteorology and Climatology, vol. 48, no. 3, pp. 680689, 2009.

[12] Y. Zhao, B. Wang, and Y. Wang, "Initialization and simulation of a landfalling typhoon using a variational bogus mapped data assimilation (BMDA)," Meteorology and Atmospheric Physics, vol. 98, no. 3-4, pp. 269-282, 2007.

[13] K.-H. Chou and C.-C. Wu, "Typhoon initialization in a mesoscale combination of the bogused vortex and the dropwindsonde data in DOTSTAR," Monthly Weather Review, vol. 136, no. 3, pp. 865-879, 2008.

[14] F. Weng, "Advances in radiative transfer modeling in support o satellite data assimilation," Journal of the Atmospheric Sciences, vol. 64, no. 11, pp. 3799-3807, 2007.

[15] X. Zou, Q. Xiao, A. E. Lipton, and G. D. Modica, "A numerical study of the effect of GOES sounder cloud-cleared brightness 
temperatures on the prediction of Hurricane Felix," Journal of Applied Meteorology, vol. 40, no. 1, pp. 34-55, 2001.

[16] J. F. Le Marshall, L. M. Leslie, R. F. Abbey Jr., and L. Qi, "Tropical cyclone track and intensity prediction: the generation and assimilation of high-density, satellite-derived data," Meteorology and Atmospheric Physics, vol. 80, no. 1-4, pp. 43-57, 2002.

[17] Y.-F. Wang, B. Wang, G. Ma, and Y.-S. Wang, "Effects of 4DVAR with multifold observed data on the typhoon track forecast," Chinese Science Bulletin, vol. 48, pp. 93-98, 2003.

[18] H. Zhang, J. Xue, G. Zhu, S. Zhuang, X. Wu, and F. Zhang, "Application of direct assimilation of ATOVS microwave radiances to typhoon track prediction," Advances in Atmospheric Sciences, vol. 21, no. 2, pp. 283-290, 2004.

[19] T. H. Zapotocny, J. A. Jung, J. F. Le Marshall, and R. E. Treadon, "A two-season impact study of four satellite data types and rawinsonde data in the NCEP global data assimilation system," Weather and Forecasting, vol. 23, no. 1, pp. 80-100, 2008.

[20] G. A. Grell, J. Dudhia, and D. R. Stauffer, "A description of the fifth-generation penn state/NCAR Mesoscale Model (MM5)," NCAR Technical Note NCAR/TN-398 + STR, National Center for Atmospheric Research, Boulder, Colo, USA, 1994.

[21] I. B. Troen and L. Mahrt, "A simple model of the atmospheric boundary layer; sensitivity to surface evaporation," BoundaryLayer Meteorology, vol. 37, no. 1-2, pp. 129-148, 1986.

[22] J. R. Eyre and H. M. Woolf, "Transmittance of atmospheric gases in the microwave region: a fast model," Applied Optics, vol. 27, pp. 3244-3249, 1988. 

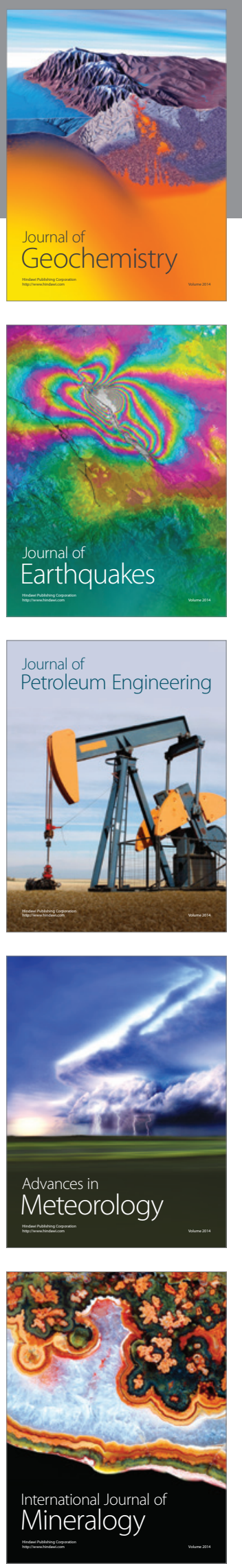
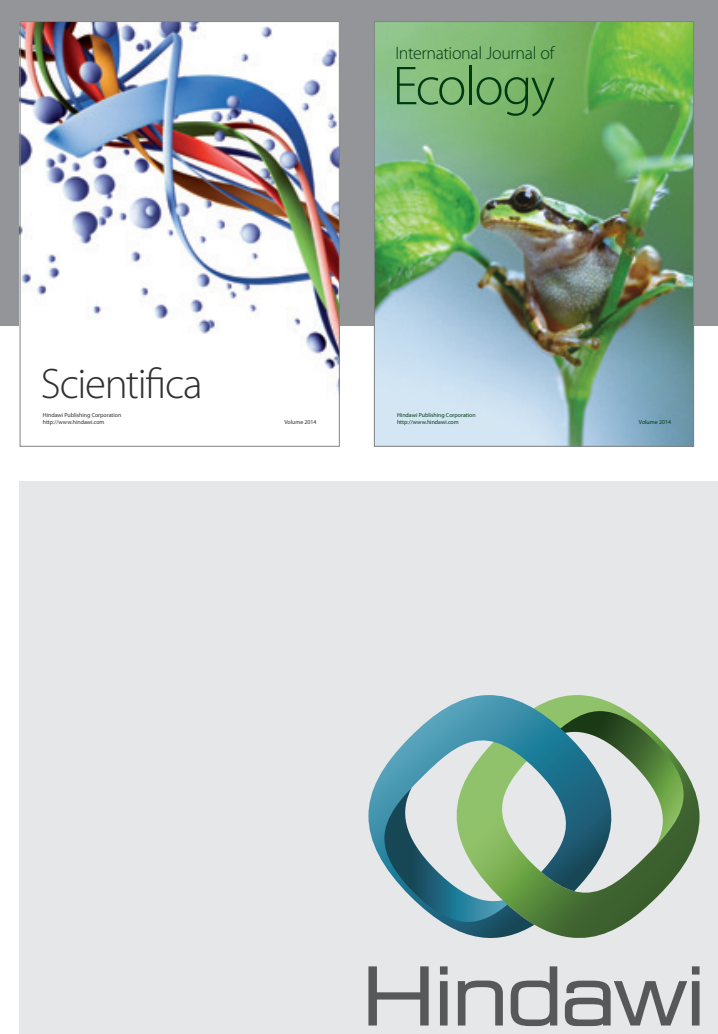

Submit your manuscripts at http://www.hindawi.com
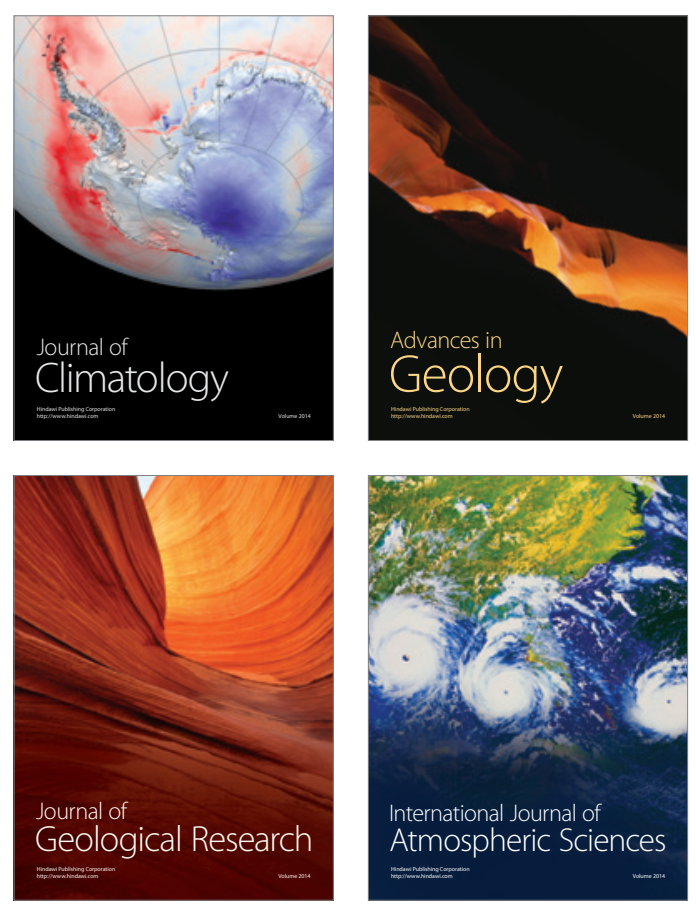
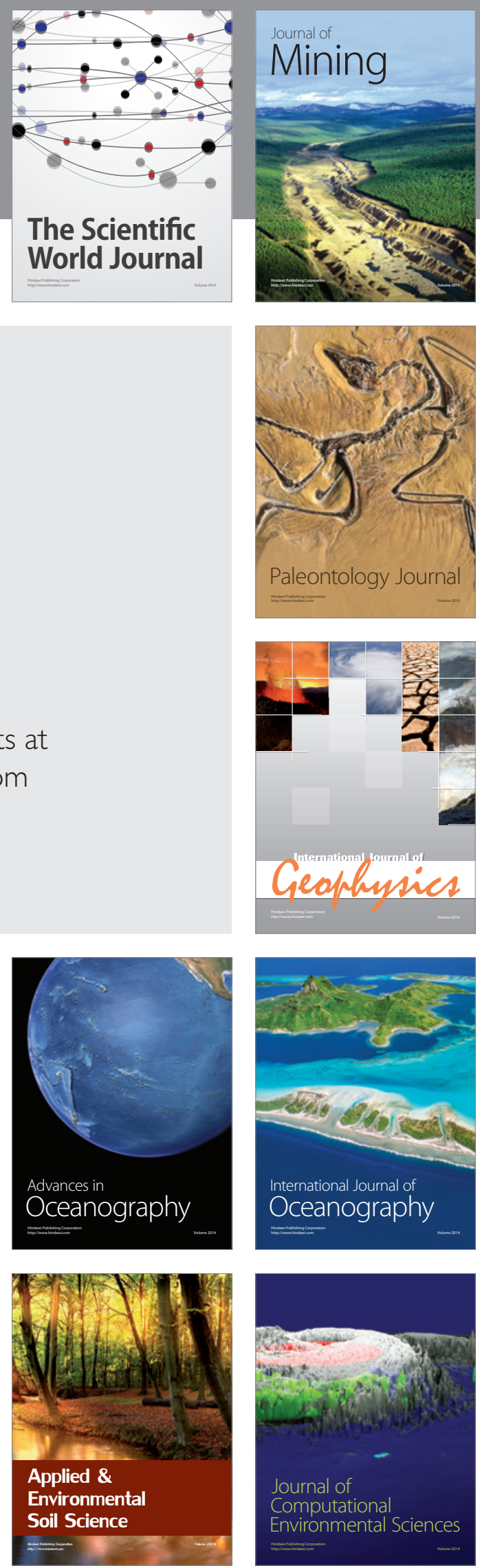